\title{
Single neurons needed for brain asymmetry studies
}

\author{
Athina Samara * \\ Department of Physiology, Institute of Basic Medical Sciences, University of Oslo, Oslo, Norway \\ ${ }^{*}$ Correspondence: athina.samara@medisin.uio.no \\ Edited by: \\ Yannis Karamanos, Université d'Artois, France
}

Keywords: RNA-seq, asymmetry, neurons, proteomics, genomics, heterogeneity, single-neuron diversity

The complexity of the human brain is inconceivable, and can only be conveyed in numbers; for example, the 100 billion neurons comprising it, without taking into account the numerous non-neuronal cells i.e., the glia and the astrocytes. Each neuron may communicate with another 10,000 neurons, with trillions of intercellular junctions (synapses) established by adulthood; synapses demonstrating plasticity and prone to change over time. Human genome sequences foresee that each neuron could express over 20,000 protein coding genes and thousands of non-coding RNAs. Recent advances in high-throughput techniques such as RNASeq, proteomics, bioinformatics and metaanalyses of the resulting data, unveil deeper brain heterogeneity than what researchers had suspected.

Brain asymmetry in terms of neuronal heterogeneity, makes the examination of the connections and biochemical steady state of each neuron seemingly insurmountable. The Allen Institute for Brain Science (http://www.alleninstitute.org/) educates naïve minds and experts further: neuronal vs. non-neuronal populations, heterogeneity due to developmentally regulated specific gene expression in different brain regions to attain brain maturation. Scrutinizing the highly polarized nature of neurons being responsible for uneven distribution of the gene products; thus compartments such as soma, axon, dendrites and synapses contain unique pools of RNAs and proteins and may have different signaling networks.

Earlier single neuron transcriptome studies in Caenorhabditis elegans demonstrated that even functionally related neurons express $>1000$ genes in a differential manner (Etchberger et al., 2007). Given that the transcriptome of each single neuron undertakes dynamic changes in response to external and developmental signals, single neuron RNA-Seq can give undiluted cell-specific information, compared to pooling groups of neurons. Single-cell genome analysis is inherently challenging, and RNA-Seq does not depend on genome annotation for prior probe selection; these differences with microarrays lower RNA-Seq bias. Both RNA-Seq and microarrays depend on genome amplification (cDNA) before measurement, hence validation is impossible because the single-cell's genome before amplification cannot be determined [for neuron specific genomics proteomics reviews see Hebenstreit et al. (2012); Kadakkuzha and Puthanveettil (2013)]. According to the work of Qiu et al. (2012), electrophysiology, and single mouse neuron microaspiration gave $\sim 10 \mathrm{pg}$ of RNA both in brain slices or 12DIV neuronal cultures. RNA-Seq confirmed that morphologically identical mouse neurons, even from the same brain region, have distinct expression patterns. Their data suggested that the observed differences among different single neuron analyses of gene expression are mostly due to cell-to-cell phenotypic variability rather than noise due to technical issues. Undoubtedly, the mRNA content residing in distal compartments (e.g., axons, dendrites) might not be captured by microaspiration (Eberwine, 2001; Eberwine et al., 2001; Batish et al., 2012). Qiu et al advised laser capture microdissection as an alternative method to single-cell aspiration for single cell studies.

Single neuron transcriptomics optimization methods are currently under scrutiny. But the full molecular signature of a neuron goes beyond genomics and transcriptomics, as it embraces the proteome. Changes in the proteome are intricate, not only by the decrease or increase in the number of copies of protein molecules of interest, but also by changes in the functional states of the proteins; states such as phosphorylation, glycosylation etc, regulated by their association with other proteins and protein modifications. A single-cell functional proteomics assay measures the quantity of such a functional state of a given protein or panel of proteins and the changes in a cell's activity (such as neuronal migration) or activation/inhibition of protein signaling networks. [A recent review by Wei et al covers emerging technologies and associated applications, designed to characterize cellular heterogeneity by single-cell functional proteomics (Wei et al., 2013)].

The proteome of cells between different brain regions is known to differ greatly, giving rise to a multitude of studies of brain asymmetry. However, unlike detecting low copies of RNAs by linear amplification, there are no amplification methodologies for proteins, thus low abundance proteins may be lost during sample analysis [for methods in neuroproteomics (Craft et al., 2013)].

A series of emerging technologies enabled the examination of changes in proteome at the single-cell level. Studies from the Sweedler group bridging chemistry and neurobiology, have established the use of mass spectrometry-based approaches to perform micro chemical analysis of single neurons, studying neuropeptides and even metabolomics (Bai et al., 2011; Rubakhin et al., 2011; Yin et al., 2011; Ahmed et al., 2012; Cecala et al., 2012; Lanni et al., 2012; Nemes et al., 2012; Neupert et al., 2012; Tucker et al., 2012; Rubakhin et al., 2013). The group develops mass spectrometry protocols to measure peptides in individual neurons and individual neuronal processes, and they aspire to develop a low-concentration 
assay detecting peptides from subcellular microenvironments such as fractions of single cells.

Proteins from single cells can bind to antibodies coupled with fluorescence, and these antibodies may be spotted and arrayed on a chip in order to profile intracellular pathways (Shi et al., 2012). Thus, the foundations for different antibody arrays for single neuron specific pathways are set. A multidisciplinary, multimillionpound Imperial College (UK) project, entitled "Single Cell Analysis" where David Klug is the Co-Director promotes research in this field. A study by Klug's group (Salehi-Reyhani et al., 2011) presented the development of an all-optical platform to analyze protein copy numbers in single cells, via microfluidic antibody capture detecting proteins using Total Internal Reflection Fluorescence (TIRF) microscopy. This methodology is particularly effective in studies of genetically labeled proteins or protein complexes in single cells. Willison and Klug advanced this to the use of the MAC chip; a "label free microfluidic antibody capture chip platform to quantify copy numbers of many proteins from a single cell in multiplexed single assay format" (Willison and Klug, 2013).

Another mind-blowing study describes the Nano needle biosensor that can be injected in living cells (Esfandyarpour et al., 2013a,b). The total diameter of the sensor is about $100 \mathrm{~nm}$, and key element of this device is a $10 \mathrm{~nm}$ wide annular gap at the end of the needle. Any change in the population of molecules in this gap would result in a change of impedance across the gap, where single molecule detection should be possible. Flowing the solution containing the target molecules over arrays of such structures, where each one has its own integrated circuitry, "real-time" detection (for several minutes) of label free molecules can be attained without sacrificing sensitivity. The study concludes that the fully developed sensor would directly monitor protein quantities within a single living cell.

In conclusion, although the morphological diversity of neuronal cell types is a century old known secret, understanding the molecular complexity of terminally differentiated neurons has been achieved in the last years. The methodological advancements toward complete single neuron signature come from a series of science branches, unveiling the secrecy neuron by neuron. A transparent mouse brain or even a human brain slice, labeled with fluorescent mRNA or protein probes, or even stained for different neuronal circuits, plus the tools to isolate each one for single cell transcriptomics or proteomics studies, was in the realm of science fiction till last year. Now we can start dreaming big: dreaming of live transparent brain slices, where single neurons fluorescent for the mRNAs of choice could be analyzed for their proteome changes. We could design experiments with live transparent brain slices injected with Nano sensors that monitor proteome and transcriptome at real time. We could go further to work with CLARITY transparent Brainbow whole animal experiments (Livet et al., 2007; Chung and Deisseroth, 2013). We would then start to grasp the finer details regarding neurons; how they decide when and how to follow different developmental and environmental cues, how they pick the neurons they communicate with, what changes according to sex or hemisphere positioning, what changes happen in the diseased cells and how all these translate into cognition and behavior. Processing all these data of the asymmetrical-neuron studies will finally carve out the asymmetrical human brain. We might be lucky enough to see it realized in our times.

\section{REFERENCES}

Ahmed, W. W., Li, T. C., Rubakhin, S. S., Chiba, A. Sweedler, J. V., and Saif, T. A. (2012). Mechanical tension modulates local and global vesicle dynamics in neurons. Cell. Mol. Bioeng. 5, 155-164. doi: 10.1007/s12195-012-0223-1

Bai, L., Romanova, E. V., and Sweedler, J. V. (2011). Distinguishing endogenous D-amino acidcontaining neuropeptides in individual neurons using tandem mass spectrometry. Anal. Chem. 83, 2794-2800. doi: 10.1021/ac20ssss0142m

Batish, M., van den Bogaard, P., Kramer, F. R., and Tyagi, S. (2012). Neuronal mRNAs travel singly into dendrites. Proc. Natl. Acad. Sci. U.S.A. 109, 4645-4650. doi: 10.1073/pnas.1111226109

Cecala, C., Rubakhin, S. S., Mitchell, J. W., Gillette, M. U., and Sweedler, J. V. (2012). A hyphenated optical trap capillary electrophoresis laser induced native fluorescence system for single-cell chemical analysis. Analyst 137, 2965-2972. doi: 10.1039/c2an35198f

Chung, K., and Deisseroth, K. (2013). CLARITY for mapping the nervous system. Nat. Methods 10, 508-513. doi: 10.1038/nmeth.2481.
Craft, G. E., Chen, A., and Nairn, A. C. (2013). Recent advances in quantitative neuroproteomics. Methods 61, 186-218. doi: 10.1016/j.ymeth.2013.04.008

Eberwine, J. (2001). Molecular biology of axons: "a turning point.... Neuron 32, 959-960. doi: 10. 1016/S0896-6273(01)00555-4

Eberwine, J., Miyashiro, K., Kacharmina, J. E., and Job, C. (2001). Local translation of classes of mRNAs that are targeted to neuronal dendrites. Proc. Natl. Acad. Sci. U.S.A. 98, 7080-7085. doi: 10.1073/pnas.121146698

Esfandyarpour, R., Esfandyarpour, H., Harris, J. S., and Davis, R. W. (2013a). Simulation and fabrication of a new novel 3D injectable biosensor for high throughput genomics and proteomics in a lab-on-a-chip device. Nanotechnology 24, 465301. doi: 10.1088/0957-4484/24/46/465301

Esfandyarpour, R., Esfandyarpour, H., Javanmard, M., Harris, J. S., and Davis, R. W. (2013b). Microneedle biosensor: a method for direct labelfree real time protein detection. Sens. Actuators B Chem. 177, 848-855. doi: 10.1016/j.snb.2012. 11.064

Etchberger, J. F., Lorch, A., Sleumer, M. C., Zapf, R., Jones, S. J., Marra, M. A., et al. (2007). The molecular signature and cis-regulatory architecture of a C. elegans gustatory neuron. Genes Dev. 21, 1653-1674. doi: 10.1101/gad.1560107

Hebenstreit, D., Deonarine, A., Babu, M. M., and Teichmann, S. A. (2012). Duel of the fates: the role of transcriptional circuits and noise in CD4+ cells. Curr. Opin. Cell Biol. 24, 350-358. doi: 10.1016/j.ceb.2012.03.007

Kadakkuzha, B. M., and Puthanveettil. S. V. (2013). Genomics and proteomics in solving brain complexity. Mol. Biosyst. 9, 1807-1821. doi: $10.1039 / \mathrm{c} 3 \mathrm{mb} 25391 \mathrm{k}$

Lanni, E. J., Rubakhin, S. S., and Sweedler, J. V. (2012). Mass spectrometry imaging and profiling of single cells. J. Proteomics 75, 5036-5051. doi: 10.1016/j.jprot.2012.03.017

Livet, J., Weissman, T. A., Kang, H., Draft, R. W., Lu, J., Bennis, R. A., et al. (2007). Transgenic strategies for combinatorial expression of fluorescent proteins in the nervous system. Nature 450, 56-62. doi: 10.1038/nature06293

Nemes, P., Knolhoff, A. M., Rubakhin, S. S., and Sweedler, J. V. (2012). Single-cell metabolomics: changes in the metabolome of freshly isolated and cultured neurons. ACS Chem. Neurosci. 3, 782-792. doi: 10.1021/cn300100u

Neupert, S., Rubakhin, S. S., and Sweedler, J. V. (2012). Targeted single-cell microchemical analysis: MS-based peptidomics of individual paraformaldehyde-fixed and immunolabeled neurons. Chem. Biol. 19, 1010-1019. doi: 10.1016/j.chembiol.2012.05.023

Qiu, S., Luo, S., Evgrafov, O., Li, R., Schroth, G. P., Levitt, P., et al. (2012). Single-neuron RNA-Seq: technical feasibility and reproducibility. Front. Genet. 3:124. doi: 10.3389/fgene.2012. 00124

Rubakhin, S. S., Lanni, E. J., and Sweedler, J. V. (2013). Progress toward single cell metabolomics. Curr. Opin. Biotechnol. 24, 95-104. doi: 10.1016/j.copbio.2012.10.021

Rubakhin, S. S., Romanova, E. V., Nemes, P., and Sweedler, J. V. (2011). Profiling metabolites and 
peptides in single cells. Nat. Methods 8(4 Suppl.), S20-S29. doi: 10.1038/nmeth.1549

Salehi-Reyhani, A., Kaplinsky, J., Burgin, E., Novakova, M., deMello, A. J., Templer, R. H., et al. (2011). A first step towards practical single cell proteomics: a microfluidic antibody capture chip with TIRF detection. Lab Chip 11, 1256-1261. doi: $10.1039 / \mathrm{c} 0 \mathrm{lc} 00613 \mathrm{k}$

Shi, Q., Qin, L., Wei, W., Geng, F., Fan, R., Shin, Y. S., et al. (2012). Single-cell proteomic chip for profiling intracellular signaling pathways in single tumor cells. Proc. Natl. Acad. Sci. U.S.A. 109, 419-424. doi: 10.1073/pnas.11108 65109

Tucker, K. R., Li, Z., Rubakhin, S. S., and Sweedler, J. V. (2012). Secondary ion mass spectrometry imaging of molecular distributions in cultured neurons and their processes: comparative analysis of sample preparation. J. Am. Soc. Mass. Spectrom. 23, 1931-1938. doi: 10.1007/s13361-012-0472-1

Wei, W., Shin, Y. S., Ma, C., Wang, J., Elitas, M., Fan, R., et al. (2013). Microchip platforms for multiplex single-cell functional proteomics with applications to immunology and cancer research. Genome Med. 5, 75. doi: 10.1186/gm479

Willison, K. R., and Klug, D. R. (2013). Quantitative single cell and single molecule proteomics for clinical studies. Curr. Opin. Biotechnol. 24, 745-751. doi: 10.1016/j.copbio.2013.06.001

Yin, P., Hou, X., Romanova, E. V., and Sweedler, J. V. (2011). Neuropeptidomics: mass spectrometrybased qualitative and quantitative analysis. Methods Mol. Biol. 789, 223-236. doi: 10.1007/9781-61779-310-3_14
Received: 02 December 2013; accepted: 21 December 2013; published online: 16 January 2014.

Citation: Samara A (2014) Single neurons needed for brain asymmetry studies. Front. Genet. 4:311. doi: 10.3389/fgene.2013.00311

This article was submitted to Neurogenomics, a section of the journal Frontiers in Genetics.

Copyright (c) 2014 Samara. This is an open-access article distributed under the terms of the Creative Commons Attribution License (CC BY). The use, distribution or reproduction in other forums is permitted, provided the original author(s) or licensor are credited and that the original publication in this journal is cited, in accordance with accepted academic practice. No use, distribution or reproduction is permitted which does not comply with these terms. 